\title{
MISCELÁNEA
}

\section{EL TOPÓNIMO FANDAQ EN LAS TAHAS DE FERREYRA, POQUEYRA Y XUBILES}

EN 1527

1.0 El libro de habices de 1527 de las tahas de Ferreyra, Poqueyra y Xubiles ha sido ya objeto de mi atención y estudio ${ }^{1}$, pero el repertorio de mil treinta topónimos menores que dicho manuscrito inédito contiene precisa un estudio detallado, haciendo pequeñas monografías sobre cada tipo, en este sentido ya he iniciado esta tarea con el estudio del topónimo hā ra 'barrio', 'caserío', que se presenta en ciento un ejemplos ${ }^{2}$. Ahora considero interesante someter a revisión el topónimo j a n d a q, con una ya señalada representación en la Toponimia peninsular, AsfN ${ }^{3}$, VRRNET $^{4}$, pues así vamos a encontrar cincuenta y dos representantes de dicho topónimo, situados en una zona de las Alpujarras, que nos van a ofrecer informaciones muy valiosas sobre configuración del terreno, vegetación, oficios, y que nos permiten en cierto modo reconstruir el ambiente socio-económico del final del siglo Xv,

1 Juan Martinez Ruiz, «Arabismos y mozarabismos en el 'Libro de los Habizes de las tahas de Ferreyra, Poqueyra y Xubiles» (año 1527), en RFE, LIX, 1977 (1979), págs. 297-308; ídem, «Toponimia menor de las tahas de Ferreyra, Poqueyra y Xubiles, según el libro manuscrito inédito de Habices de 1527». Comunicación al XVI Congreso Internacional de Lingüística y Filología Románicas, Palma de Mallorca, 7-12 abril de 1980, en prensa, en las Actas correspondientes.

2 Juan Martinez Ruiz, «El topónimo $\mathrm{h} \bar{a} \mathrm{r}$ a en las tahas de Ferreyra, Poqueyra y Xubiles en 1527», en Estudios dedicados a Manuel Alvar, en prensa.

3 Miguri asin Palacios, Contribución a la Toponimia árabe en España, 2.^ ed., Madrid, 1944.

4 Juan Vernet, «Toponimia Arábiga», en Enciclopedia Lingülstica Hispánica, t. I, Madrid, 1959, págs. $561-578+4$ mapas.

LXII. -6 
antes de la repoblación castellana y de la ruina total de la toponimia patrimonial, según puede verse en las modernas cartografías de dicha zona.

1.1 Ante todo conviene señalar la zona de dispersión del topónimo según el orden señalado por el manuscrito inédito del Archivo de la Curia Eclesiástica de Granada, titulado aLibro de apeamiento de los habizes del Alpuxarra, de las tahas de Ferreyra, Poqueyra y Xubiles. Año 1527».

En primer lugar figura Pooueyra, que comprende cuatro zonas:

1 Capileyra, 2 Bubión de Capileyra, 3 Beniozmin y 4 Pampaneyra.

La taha de FerReYRA comprende:

1 Pitras, 2 Capileyra de Ferreyra, 3 Fondales, 4 Mecina, 5 Ferreyrola, 6 Pórtugos, 7 Haratalbeytar.

La taha de XUBILBS comprende:

1 Trevélez, 2 Notáez, 3 Xubiles Capileyra, 4 Cástaras, 5 Nieles, 6 Lobras,

7 Timen, 8 Cádiar, 9 Narila, 10 Bérchul, 11 Alcuta, 12 Cuxorio, 13 Purchenas, 14 Mecina, 15 Godco, 16 Yátor, 17 Yegen y 18 Válor.

En Poqueyra el topónimo j a n d a q se distribuye así:

al-Handac aljuiza, 12v, 9-10; Alhandac aljuliza, en Poqueira Capileyra.

Handac barceical, 12v, 13-14, en Poqueyra Capileyra.

En FerReyra encontramos:

a) en Pitras:

Handac alauc, 47v, 32; Handac cabar, 54r, 32-33.

Handac alfadid, 52v, 17.

Handac alhajuza, 49r, 14, 29, 38; Handac alfahuza, 49r, 21.

Handac alhara, 53r, 12.

Alhadec alfaray, 53r, 24.

Handac almaçera, «barranco que se dizex, 46v, 10.

Handac almali, 52v, 9.

Alhandac almozaguar, 52v, 45.

b) en Capileyra:

Handac, pago, 69v, 20.

Handac adaya, pago, 58r, 7.

Handac alhajuza, 56r, 23, 25.

Handac alzugaya, 57r, 12.

Alhandac mundir, pago, 57v, 19.

Handaca xecril, 61r, 26.

Handaca, 60r, 1-2. 
c) en Pórtugos:

Handac aljeuz, pago, 109v, 7.

d) en Haratalbeytar:

Handac aldar, 113v, 5.

Handac April, 116v, 26.

En Xubiles los representantes de $\mathbf{j}$ a $\mathrm{n}$ d a $q$ son:

a) en Cástaras:

Handac alacmar, pago, 160r, 31.

Handac aljoayza, 152v, 2.

Handac almoral, pago, 157r, 23.

Handac alnajar, 161r, 11, 1415.

Handac mazaupa, 156r, 13; Handac Abenhauz, 163r, 2.

b) en Notáez:

Handac almaçaba, 132v, 15, 18-19.

Handac alqeqela, 132v, 22-23.

Handac cafar, 133v, 5.

Handac niles, 132r, 29.

c) en Mecina:

Handac alfex, rábita de, 249v, 9.

Handac bacatel, pago, 259r, 7, 13.

Alhandic, pago, 250v, 24.

d) en Cádiar:

Handac alcadin, 194r, 12.

Handac xaybin, 200r, 12; 201r, 18; Alhandac, 193r, 24.

e) en Yátor:

Handac alharraz, haca, 267v, 4.

Alhandac, pago, 268r, 22-23.

f) en Lobras:

Alhandac, 177v, 17.

Handac Abeauz, 182v, 24, 25.

Handac Abehauf, 176r, 24.

8) en Trevélez:

Handac alguadixi, 123v, 1.

Alhandac, pago, 140v, 30.

h) en Capileyra:

Handac alhox, "barranco que se dize», 144r, 2425.

Alhandac, 141r, 1, 18; 143v, 12, 21.

i) en Cuxorio:

Handac almex, 231v, 17.

Handac, pago, 235v, 20, 28. 
j) en Yegen:

Handac aladira, 276v, 20-21.

Handac algeuza, pago, 277v, 2425.

k) en Purchenas:

Alhandac, 241r, 18-19.

1) en Timen:

Alhandac, 186r, 14.

m) en Válor:

Handac maçarra, «barranco que dizen», 300v, 29-30.

El siguiente cuadro nos permite contemplar la densidad del topónimo $\mathrm{j}$ a n d a $\mathrm{q}$ en las tres tahas alpujarreñas:

\begin{tabular}{|c|c|c|c|}
\hline \multirow[t]{3}{*}{ POOURYRA } & & & TOTAL \\
\hline & Capileyra & 2 & 2 \\
\hline & Pitras & 9 & \\
\hline \multirow{11}{*}{ Ferreryra } & & & \\
\hline & Capileyra & 7 & \\
\hline & Haratalbeytar & 2 & \\
\hline & Portugos & 1 & \\
\hline & & 19 & 19 \\
\hline & Castaras & 6 & \\
\hline & Notbez & 4 & \\
\hline & Mecina & 3 & \\
\hline & Lobras & 3 & \\
\hline & Cádiar & 2 & \\
\hline & Yátor & 2 & \\
\hline \multirow[t]{9}{*}{ XubIubs } & & & \\
\hline & Trevallez & 2 & \\
\hline & Capileyra & 2 & \\
\hline & Cuxorio & 2 & \\
\hline & Yegen & 2 & \\
\hline & Purchenas & 1 & \\
\hline & Timen & 1 & \\
\hline & Válor & 1 & 31 \\
\hline & & 31 & 52 \\
\hline
\end{tabular}

1.2 Antes del estudio de los tipos de $\mathrm{j}$ a n d a $\mathrm{q}$ en la citada zona alpujarreña, sería muy ilustrativo observar las variedades que ofrece en todos los territorios de que disponemos información. 
En primer lugar notamos la falta del término en el Líbano WiLd ${ }^{3}$, es frecuente en el norte de Africa, en Marruecos $^{6}$ en el territorio de Yebăla, he señalado:

El jandaq, Anyera, El Barqöqyēn.

Jandaq 'ašiš 'barranco de los nidos', Benī Ider, Saylūla.

Jandaq el senān 'barranco de los dientes', Benī Meșawar, El Jayt el Sefli.

Jandaq el kebš 'barranco del carnero', Wadrās, El Bakkara.

El Jandaqyēn 'los dos barrancos', Benī Ider, Saylūla.

El Jenädeq 'los barrancos', Benī Meşawar, El Hayt el Foqi.

En España, Asł́ ${ }^{7}$ dio a conocer Aljandaque en Málaga, arroyo en el término rural de Alpendeire, Alfandeche en Valencia, Alfándiga en Lugo, Alfántega en Huesca y Alhándiga en Málaga, en todos ellos el término árabe a l-j a n d a q se traduce por 'barranco', 'foso'.

En Sicilia, Pellegrini ${ }^{8}$ reúne una notable representación del topónimo sacada de IDRIsI ${ }^{9}$, de CUSA ${ }^{10}$, también ofrece ejemplos de Malta según AQuilina ${ }^{11}$ :

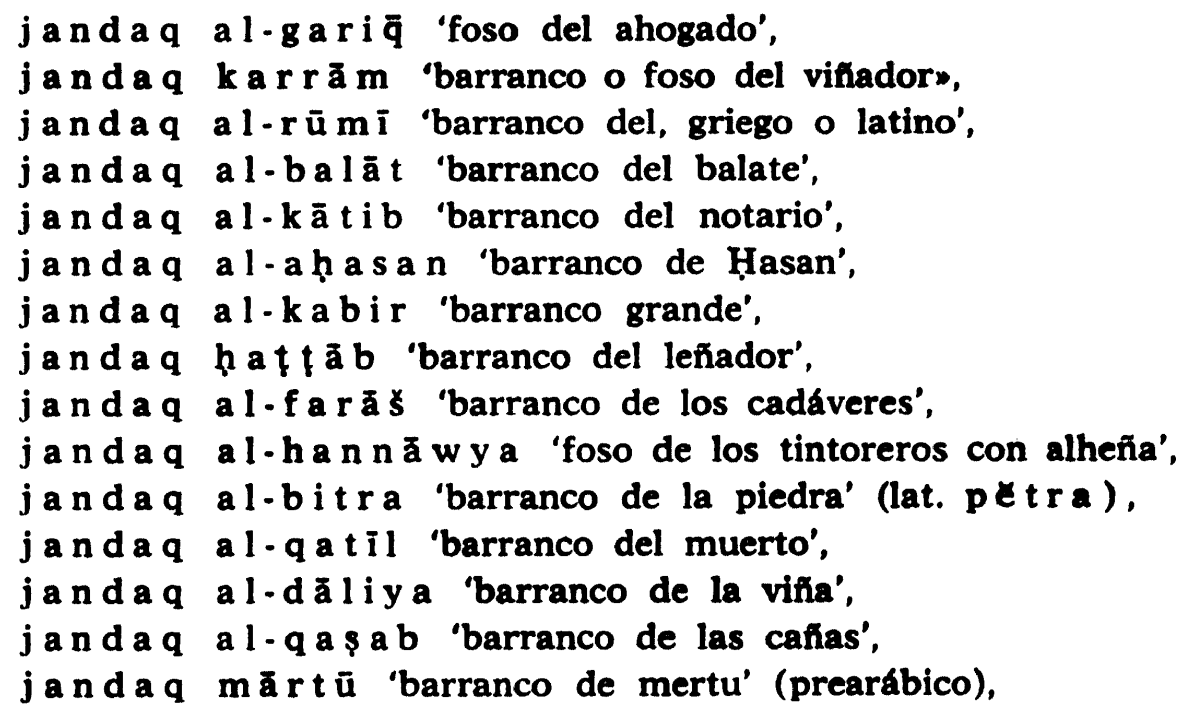

5 Stepan Wrud, Libanesische Ortsnamen, Typologie und Deutung, Beiruter Texte und Studien herausgegeben vom Orient-Institut der deutschen morgenländischen Gesellschaft, Band. 9, Beirut, 1973.

6 Juan Martinez Ruiz, "Toponimia menor de Pebăla (Marruecos)», en Cuadernos de la Biblioteca Española de Tetuán, núms. 19-20, 1979, págs. 23-49.

7 Ob. cit., en nota 3.

- Grovan Battista Pzllegrini, Gli arabismi nelle lingue neolatine con speciale riguardo all'Italia, 2 vols., Brecia, 1972.

- AL-IDRIsI, Il libro di Ruggero, tradotto e annotato da U. Rizzitano, Palermo (Flacovio), 1966.

10 Salvatore J. Cusa, Diplomi Greci ed Arabi di Sicilia, pubblicati nel testo originale, tradotti e illustrati, Palermo, 1868-1882.

11 J. Aqunlna, Papers in Maltese linguistics, Royal University of Malta, 1961, y The structure of Maltese. A study in mixed grammar and vocabulary, Malta, 1959. 
jandaq bu-Ș a daqa 'barranco de Abu-Şadaqa',

ja ndaq ša raf 'barranco del otero',

jand a a l-y a hüd 'barranco de los judíos',

jandaq balunbū 'barranco de la paloma' (del latín palumba),

ja n da q a l-kandār 'barranco del incienso',

ja ndaq a l-šay ḩ 'barranco del anciano',

ja ndaq a l-silā h 'barranco de las armas',

ja ndaq al-gulām 'barranco del joven, del siervo',

jandaq $\$$ ant Abrāqā t 'barranco o foso de San Brancato',

jandaq a l-a huwā 'barranco de los abrevaderos, de los estanques',

jandaq a l-baqari 'barranco del buey',

jandaq al-marr 'barranco del pico de hierro o del paso'.

En esta relación he seleccionado los ejemplos que más parecido puedan tener con los topónimos alpujarreños, como podemos comprobar figuran oficios, características del terreno, vegetación, onomástica, animales... grupos análogos podemos establecer con los topónimos contenidos en el libro de habices de 1527.

2.1 El primer grupo, el más numeroso está formado por 13 nombres de barrancos referente a términos de agricultura y fitoponimia:

Handac alfex, ár. ja nda q a l-f a ḥ ș, en Xubiles Mecina. Según Yãqūt *Todo lugar poblado, sea llano o montańoso, a condición de que esté cultivado, se llama $f$ a h $\$ \times 12$.

Handac algeuza, pago, ár. ja nda q a l- a a za 'barranco del nogal', Freytag, I, pág. 324 13. En Xubiles Yegen.

Handac alhara, ár. jandaq a l- $\$$ a ' $\mathrm{ra}$ 'barranco de la jara', en Ferreyra Pitras. Figura en Asto 14 La Jara.

Handac alhox, «barranco que se dizex, ár. ja nd a q a l- h u u sarranco del huerto, letrina', FreYtag, I, pág. 382 15. En Xubiles Capileyra.

Handac aljeuz, pago, ár. ja n d a q a l-y a w z 'barranco del nogal'. En Ferreyra Pórtugos. Freytag, I, pág. 32415.

Handac aljoayza, ár. ja nda q a l-9 u a yza 'barranco del nogalillo', en Xubiles Cástaras.

Alhandac aljuiza y Alhandac aljuliza en Poqueyra Capileyra, son grafías más o menos acertadas del topónimo anterior.

Handac almaçaba, ár. j a nd a q a l-mā s a b (del verbo 'āş a ba ) 'barranco desprovisto de hierbas y de todo pasto', FreytaG, I, pág. 3515. Rn Ferreyra Pitras.

12 GAMAL 'ABD AL-KARIM, Terminologia geografico-administrativa e Historia politico-cultural de al-Andalus en el «Mu'Dam al-buldān» de Yāqūt, Universidad de Sevilla, 1972.

13 Grorg Wilheim Freytag, Lexicon Arabico-Latinum, 4 vols., Halle, 1830-37. Vol. I, pág. 324.

14 Migurl Astn Palactos, Contribución a la Toponimia drabe de España, 2.a ed., Madrid-Granada, 1944, pág. 114.

15 G. W. FreytaG, ob. cit., en nota 13. 
Handac almex, ár. j a n d a q a l-ma y s 'barranco del almez'. Figura ma y s 'almez', Celtis australis, $L$. en el botánico hispanoárabe de los siglos XI-XII, AsfN 16. En Xubiles Cuxorio.

Handac almoral, ár. ja $\mathrm{ndaq}$ a l- + romance mor a l es frecuente esta toponimia híbrida arábigo romance, como el Guadamoral, arroyo en la provincia de Córdoba, afluente del Guadajoz, citado por SIMONET ${ }^{17}$. En Xubiles Cástaras.

Handac alqeqela, ár. j a ndaq a l-qāqull a 'barranco del cardamomo', Meyerhop 18, n.o 325, págs. 161-162, Stmonet 19, pág. 439. En Xubiles Notáez.

Handac niles, ár. j a n d a q $\mathrm{n}$ il + romance -es 'barranco de los añiles', en Xubiles Notáez. El árabe a l-nil, MeYerhor, n.0 249, pág. 12220.

Handac xecril, ár. ja n daq $\mathbf{s}$ a $\mathbf{k}$ ā $\mathrm{r}$ a + sufijo romance - ill u 'barranco del pequeño rincón de tierra labrada y sembrada', Beror ${ }^{21}$, del árabe sa kāra.

2.2 Siguen en orden de frecuencia los topónimos que hacen referencia a los rasgos físicos o impresiones que producen los barrancos:

Handac alfaray, ár. j a n d a a l-f a r a 'barranco de la alegria', FreyTAG, III, pág. 32722 . También se puede interpretar como 'barranco de Faray', nombre de persona que también figura en YAoOT ${ }^{23}$ como sinonimo de Guadalajara ma di n a F a r a y. Figura en Astin, pág. 10524. En Ferreyra Pitras.

Handac alacmar, ár. ja n d a q a l-ā q māa 'barranco de la luna', en Xubiles Cástaras. FrertaG, III, pág. 49625.

Handac alauc, ár. janda q a l-'a w q 'barranco de la suerte', en Ferreyra Pitras. FreYtag, III 26, pág. 244 • a w q significa también 'gallo, león, lobo, ave'.

Handac alcadin, ár. ja nd a q a l- $\mathrm{q}$ a di m 'barranco antiguo' en Xubiles Cádiar, FreytaG, III, pág. 41027.

16 Miguel Asf Palacios, Glosario de voces romances registradas por un botdnico anónimo hispanomusulmán (siglos XI-XII), Madrid-Granada, 1943, págs. 327 y 404.

17 Francisco Javier Simonet, Glosario de voces ibéricas y latinas usadas entre los mozarabes. Precedido de un estudio sobre el dialecto hispano-mozdrabe, Madrid, 1888, págs. 376-377.

18 MAX MeYerhop, Un glossaire de matière médicale composé par Maïmonide, Le Caire, 1940, págs. 161-162, n. 325.

19 F. J. Simonet, ob. cit., en nota 17.

20 M. MEYERHor, ob. cit., en nota 18.

21 J. B. BeLor, Dictionannaire al-faraed arabe-français, 18 ed., Beirut, 1964, páginas 382-383.

2 G. W. FreytaG, ob. cit., en nota 13.

23 Ytoot al-Hamawi, Kitäb mu'sam al-buldān, ed. Ferdinand WÜstenfeld, Leipzig, 1866-1870 (Jacut's geographisches Wörterbuch), s. v.

24 M. Asin, ob. cit., en nota 14.

25 G. W. FreytaG, ob. cit., en nota 13.

26 G. W. Freytag, ob. cit., en nota 13.

27 G. W. Freytag, ob. cit., en nota 13. 
Alhandac almozaguar, ár. ja nd a q a l-m uzaw war 'barranco deformado', en Ferreyra Pitras. Bewot, pág. 30223.

Handac bacatel, ár. j a nd a q b a q $\mathbf{t}$ a 11 'barranco del repecho falso', en Xubiles Mecina. El árabe b a q 'vano, falso', Frerrag, I, pág. 17320.

Respecto al ár. $t$ a 11 , plural $t a \bar{l}$ a 1 'colina', 'túmulo de tierra', 'túmulo arenoson, Freytag, I, pág. 19630 , he dado a conocer la forma tel, que no ocupa en RFE, LIX, 1977 (1979), págs. 304305 31, figura en el mismo libro de habices de 1527 que nos ocupa y precisamente en el mismo lugar de Xubiles Mecina, se nos aclara: «es un repecho que se dize en arávigo tel...», y más adelante: «Un pedao de tierra que está en un ribazo, que se dize en arávigo tel...».

Handac çabar, ár. ja ndaq ' ā $\$$ bā r 'barranco de los hielos', Frertag, II, pág. 477 32, también 'barranco del borde, del margen'. En Ferreyra Pórtugos.

Handac çafar, ár. j a nd a q $\$$ a ja r 'barranco de la peña, Frertag, II, pág. 484 33. En Xubiles Notáez. Hernandez, pág. 21534.

Alhandac mundir, ár. a l-j a n d a q $\mathrm{m}$ un ḍ ir el barranco florido, hermoso' en Ferreyra Capileyra, del árabe n a di ir 'nitido, floreciente, hermoso', FreYtaG, IV, pág. 29335.

Handac xaybin, ár. j a n d a q $\mathrm{S}^{\mathrm{a}}$ y bā n 'barranco frío nebuloso', en Xubiles Cádiar, FreytaG, II, pág. 46936.

2.3 Los topónimos también descubren aspectos de la vida del hombre, oficios, lugar de procedencia, actividades industriales:

Handac alfadid, ár. ja nda q a l-ḩaddād (en árabe granadino ha add i d) 'barranco del herrero', FreytaG, I, pág. 351 37. En Ferreyra Pitras.

Handac aljarraz, haça, ár. j a n d a q a l-j a r rāz 'barranco del zapatero', en Xubiles Yátor. FreYtag, I, pág. 47438.

Handac almaçera, ár. ja nda q a l-ma's a ra 'barranco del molino de aceite, de la almazara', en Ferreyra Pitras. Encontramos Almdsera en Valencia y Almázara en Navarra, Asł́, pág. 6739.

Handac almali, ár. ja nda q a l-maliy y 'barranco largo, prolongado', en Ferreyra Pitras, Belor, pág. 786 10.

23 J. B. Berot, ob. cit., en nota 21.

29 G. W. Freytag, ob. cit., en nota 13.

30 G. W. FreYtaG, ob. cit., en nota 13.

31 J. MARTINEZ RuIz, estudio citado en nota 1.

32 G. W. FreytaG, ob. cit., en nota 13.

33 G. W. FreytaG, ob. cit., en nota 13.

34 Consurzo V. Hernández Carrasco, «El árabe en la toponimia murciana», en Anales de la Universidad de Murcia, vol. XXXIV, Murcia, 1978, págs. 215 y 245.

35 G. W. FreytaG, ob. cit., en nota 13.

36 G. W. FreYtaG, ob. cit., en nota 13.

37 G. W. FreytaG, ob. cit., en nota 13.

38 G. W. FreytaG, ob. cit., en nota 13.

39 M. AsfN, ob. cit., en nota 14.

1 J. B. Belor, ob. cit., en nota 21. 
Handac alnajar, ár. ja nd a q a l-n a y ār 'barranco del carpintero', FreYtAG, IV, pág. 24341 . En Xubiles Cástaras.

\subsection{Edificios y construcciones ofrecen los topónimos:}

Handac adaya, ár. janda q a 1 - day a a 'barranco de la granja', en Ferreyra Capileyra. Véase en Asin 12, Aldea, Adaya, Aldaya, Daya y Deyd.

Handac aldar, ár. ja n d a q a l-d ā r 'barranco de la casa', en Ferreyra Haratalbeytar. Véase en AsłN ${ }^{43}$ Darabalez, dehesa de Toledo, Daragolefa, cortijo de Granada, Daramazán, dehesa de Toledo.

Handac alhajuza, ár. ja n d a q l- ha a y za 'barranco de la presa, del dique', en Ferreyra Pitras. En Freytag, I, pág. 347 H, h i y̆àz aRegio petris et montibus ab hominibus seclusa».

2.5 Simple designación de $\mathrm{j}$ a $\mathrm{n} \mathrm{d}$ a $\mathrm{q}$, sin más elementos descriptivos:

Alhandac, pago, ár. a 1 - j a n d a q 'el barroco, el foso', en Xubiles Yátor. Handaca, ár. ja nda q 'el barranco', en Ferreyra Capileyra.

Alhandic, ár. a l-janda q, ár. granadino al-jandi $\mathrm{q}$, por acción de la i m $\bar{a} l a$, CORRIBNTB 45 .

2.6 La hidronimia tiene su representación en:

Handac aladira, ár. j a n d a q a l- ' a d i r a 'barranco abundante de agua', en Xubiles Yegen. FreYtaG, III, págs. 118-119 46, ‘ a dir a xaqua abundavit locus».

Handac maçarra, ár. ja n d a q mā $\$$ i r r a 'barranco del agua fría', en Xubiles Válor. FreYraG, II, pág. 490, s i r r ${ }^{46}$.

2.7 Significados y origenes varios ofrecen:

Handac alzugaya, ár. j a nda q a l-zag ā y a 'barranco de la azagaya', en Ferreyra Capileyra. La palabra zaga 9 a es beréber, documentada ya en español hacia el año 1295, figura en Dozx 7 , NBuvonen 4 , CoroMINAS 9.

41 G. W. Freytag, ob. cit., en nota 13.

42 M. Asfin, ob. cit., en nota 14.

43 M. AstN, ob. cit., en nota 14.

4 G. W. FreytaG, ob. cit., en nota 13.

45 F. CORRIENTE, A grammatical sketch of the spanish arabic dialect bundle, Instituto Hispano-Arabe de Cultura, Madrid, 1977, págs. 22-24.

46 G. W. Freytag, ob. cit., en nota 13.

4 ReNE Dozy y W. H. ENGELManN, Glossaire des mots espagnols et portugais dérivés de l'arabe, 2." ed., Leyden, 1869, pág. 223.

- ERIK K. Neuvonen, Los Arabismos del espafiol en el siglo XIII, Leipzig, 1941, pág. 166.

49 J. Corominas, Diccionario Critico Etimológico de la Lengua Castellana, Madrid, 1954, 4 vols.; vol. I, pág. 346. 
Handac april, ár. ja nda q + mozárabe a pril 'barranco de abril', en Ferreyra Haratalbeytar. La palabra abril, del latín a prilis, está bien documentada en escritores árabes, como se puede ver en SImONET, pág. 250.

Handac barceical, en Poqueyra Capileyra. No identificado, de aspecto mozárabe y de origen desconocido, a juzgar con la posible relación de la raíz ba r ce con la palabra barcina, que tuvo gran difusión en el mozárabe, bárcha( $t$ ) 'haz manojo', 'haz de leña', Simoner, pág. 3451. También barchela del lat. pa r cella, documentada en mozárabe, Simoner, pág. 3452 , puede tener alguna posible relación.

Handac mazaupa, en Xubiles Cástaras, cuyo primer elemento es el árabe ja ndaq, pero de difícil identificación el término mazaupa. Existe un Mizep en Alicante, identificado por Asf́, pág. 12253 , con el árabe $m i \cdot z a b$ 'desaguie', pero el vocalismo de las palabras mazaupa y $m i z e p$ son totalmente distintos, sólo hay coincidencia consonántica.

2.8 Los patronímicos con $\mathrm{a} b \mathrm{~b} \mathrm{n}$, ár. literal $\mathrm{ib} \mathrm{n}$, han quedado reflejados en los topónimos:

Handac Abenhauz, ár. ja nda q i b $\mathrm{H}$ a wz 'barranco de ibn Hauz'. En Xubiles Cástaras.

Handac obeauz, ár. ja ndaq ib n Haw z 'barranco de ibn Hawz', en Xubiles Lobras.

Handac Abehauf, ár. ja nda q i b n H a w f 'barranco de ibn Hawf', en Xubiles Lobras.

Handac alguadixi, ár. ja n $\mathrm{d}$ a q a l-wā d i ši 'barranco del guadijeño', híbrido $w \bar{a} d i$ 'río' + Acci. En Xubiles Trevélez.

3.1 Como hemos podido apreciar, el estudio del topónimo árabe j a $\mathrm{n}$ a q ha recogido en sus perfiles fonético, morfológico y semántico datos muy significativos sobre la citada zona de las Alpujarras, pues no sólo ha conservado aspectos de la vida socio-económica y cultural de finales del siglo XV y principios del XVI, sino que también, desde el punto de vista lingüístico, ha sido un colector de palabras antiguas mozárabes, de fonética hispanoárabe granadina, de agricultura y fitotoponimia, evocando en determinados casos los grandes tratados de Agricultura hispanoárabe, de características geográficas y edafología, de oficios y actividades del hombre, de edificios y construcciones, de hidronimis y hasta de genealogía y onomástica. En este sentido se confirman una vez más las palabras de STBIGRR ${ }^{54}$, al comprobar cómo «Las ense-

so F. G. Simoner, ob. cit., en nota 17.

31 F. G. Simonet, ob. cit., en nota 17.

52 F. G. Simonet, ob. cit., en nota 17.

33 M. Asin, ob. cit., en nota 14.

34 ARANald Steiger, "Toponimia árabe de Murcia. Contribución a la Historia linguística de la Historia murciana», en Murgetana, n.o 11, 1958, pág. 11; CSIC, «Academia de Alfonso $\mathrm{X}$ el sabion, Murcia. 
ñanzas de la Lingüística se incrustan a veces como un martillo en la perspectiva histórica», los topónimos, verdaderos fósiles linguísticos contienen todo un legado de Historia.

Gracias a los libros de habices, la Toponimia menor granadina de finales del siglo XV y principios del XVI goza de una situación privilegiada. El momento de redacción de los libros de repartimiento de los habices iba a ser decisivo, todavia tuvo tiempo de salvar una toponimia patrimonial que pronto iba a ser barrida por la repoblación castellana y la implantación de una nueva forma de vida religiosa, cultural y jurídico-social.

En cuanto a la Geografía Lingüística, el topónimo j a r a, lo hemos visto representado en todo el área y zona de expansión temporal o definitiva de la civilización y cultura árabes, Oriente Medio, todo el norte de Africa, y en las zonas que podríamos llamar de la ArABIA PERDIDA, de Sicilia, Malta y Península Ibérica. Una aparente excepción, según la obra de WILD 55, representa el Líbano, donde no figura $\mathrm{j}$ a $\mathrm{ndaq}$, pero sí el árabe biq 'a 'hondonada, tierra fértil entre montañas, valle, depresión'. Pero es curioso observar que todos los topónimos compuestos con Bqã’ (forma libanesa del ár. b i q ${ }^{*}$ a) son de origen arameo ${ }^{56}$.

3.2 Desde el punto de vista lingüístico podemos señalar las siguientes observaciones fonéticas. En primer lugar los rasgos mozárabes de Handac april, con conservación de la sorda intervocálica -p-. La fonética hispanoárabe en la grafía de los topónimos se cumple en todo caso según las normas señaladas por STEIGER ${ }^{57}$ y por CORRIBNTE, sería ocioso repetirlas, tanto en el sistema vocálico como en el consonántico. La i ma la del árabe granadino la hemos visto reflejada en Handac xaybin, Handac alfadid, Alhandic.

En cuanto a la morfología, encontramos formas del diminutivo árabe: Handac aljoayza, ár. y u a y z a y topónimos hrbridos arábigoromances como Handac almoral, Handac April y posiblemente Handac barceical. En cuanto al topónimo compuesto Handac bacatel, ha sido el mismo documento el que nos ha ofrecido separado el significado de elemento tel, que unido a baca, ha permitido reconstruir el ár. b a q t a 11 'falso repecho', 'falsa colina'.

55 S. Wild, ob. cit., en nota 5.

36 S. WiLD, ob. cit., pág. 307, y ū r a 'hondonada, depresión de terreno, valle profundo», no es palabra de origen árabe y parece un préstamo a través del arameo. Así pues, según la obra de Wrid, b i qa y y u ra se reparten el significado de 'foso', 'hondonada', no existiendo el árabe ja nda q .

5 ARNAld Striger, Contribución a la fonética del hispano-drabe y de los arabismos en el ibero-románico y el siciliano, Madrid, 1932. 
En este proceso de identificación he consultado las obras de $\mathrm{ABOL}$ -

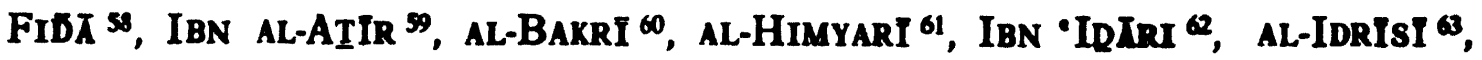
IBN JALDŪN ${ }^{64}$, AL-MÃRRAXUSI ${ }^{65}$, que han permitido conclusiones sobre la distribución geográfica del topónimo árabe ja n $\mathrm{daq}$ en el norte de Africa, y en la Península Ibérica en la época de la ocupación árabe ${ }^{66}$.

Representantes de jandaq en el libro de habices de 1527:

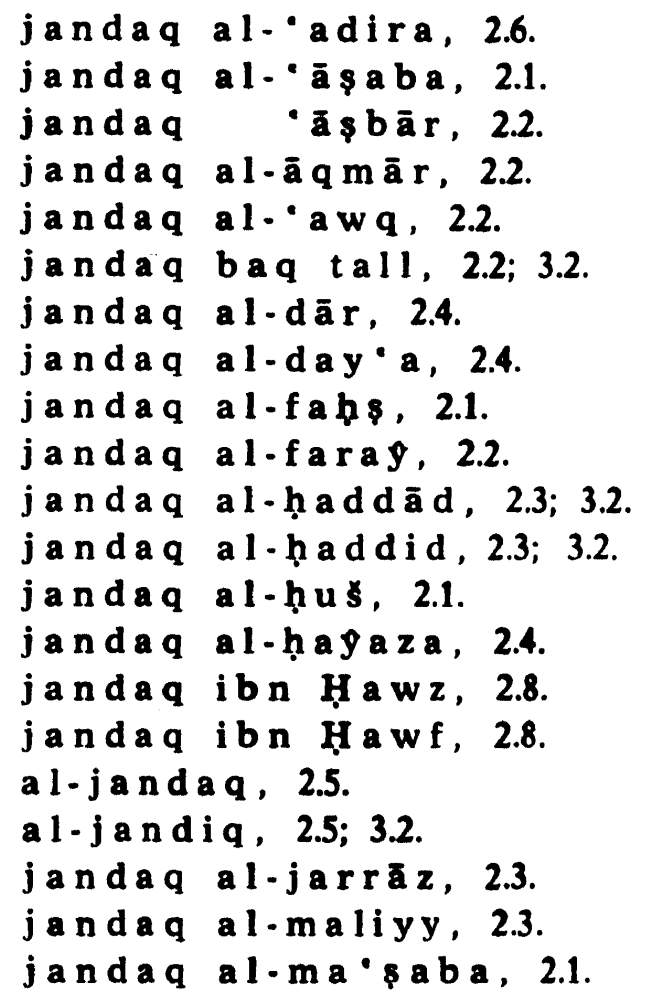

58 Asou-Fidn', Gégraphie d'Aboulféda, trad. de M. Reinguud, París, 1848.

59 Ibn Al-ATIr, Annales du Moghreb et de l'Espagne, trad. E. Pegnan, Alger, 1898.

60 Al-BaxRI, Description de l'Afrique septentrionale, trad. De Slane, Alger, 1913.

61 Al-HimyarI, trad. de E. Lévy-Provençal, La Péninsule Ibérique au Moyen Age, d'apres le «Kitab al-Rawd al-Mi'tär fi habar al-aqtar» d'Ibn Abd al-Mu'nin alHimyari, Leyden, 1938.

62 IBN - IDIRI, Histoire de l'Afrique du Nord et de l'Espagne musulmane intitulée «Kitäb al-Bayan al-Mugrib» par Ibn 'Id dari al-Marrakkuši et fragments de la Chronique de 'Arib, traduit par E. PAgnan, Argen, 1904.

63 AL-IDRIsI, Nuzhat al-mustäq, Description de l'Afrique et de l'Espagne, ed. y trad. franc. de R. Dozy y M. J. DE GozJE, Leyden, 1866. Reimpresión, Amsterdam, 1969.

4 IBN JALDON, Histoire des berbères et des dynasties musulmanes de l'Afrique septentrionale, trad. DE SLANE, París, 1925.

(o Al-MarrXxusı, Kitäb al-Mu'yib fí taljis ajbär al-Mugrib, trad. A. Huicr MrRANDA, Tetuán, 1955.

66 Quiero expresar mi agradecimiento al Catedrático y Director del Departamento de Historia del Islam de la Facultad de Filosofia y Letras de la Universidad de Granada, Dr. D. JACrNTo Boscr VILA, por las orientaciones y facilidades que en todo momento ha dado para el manejo y consulta de obras de su Departamento, así como a Profesores y personal del mismo. 


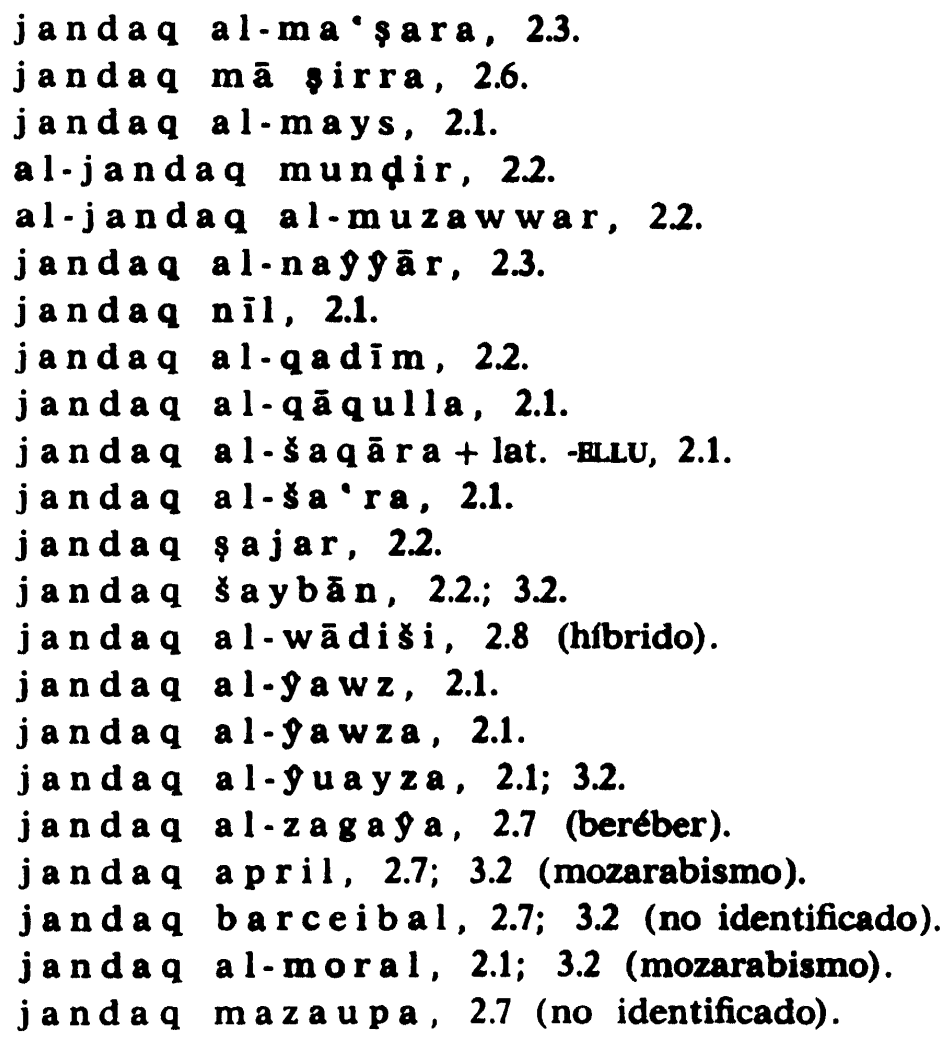

Juan Martinez Ruiz 